\title{
A General Interference-Aware Framework for Joint Routing and Link Scheduling in Wireless Mesh Networks
}

\author{
Leonardo Badia and Alessandro Erta, IMT Lucca Institute for Advanced Studies \\ Luciano Lenzini, University of Pisa \\ Michele Zorzi, University of Padova
}

\begin{abstract}
Joint design and optimization of traditionally independent problems such as routing and link scheduling have recently become one of the leading research trends in wireless mesh networks. Although technically challenging, cross-layering is, in fact, expected to bring significant benefits from the network resource exploitation standpoint to achieve high system utilization. In this article we propose a versatile framework for joint design of routing and link scheduling, introducing the notion of link activation constraints, which are related to the transceiver capability and the broadcast nature of the wireless medium. To this end, we introduce a taxonomy of wireless interference models to harmonize existing approaches presented in the literature. Finally, we evaluate the impact on network capacity of the various interference models when optimal joint routing and link scheduling are employed.
\end{abstract}

$\mathrm{n}$ a wireless mesh network (WMN) [1] end users are provided with wireless broadband connectivity by means of a predefined system hierarchy. The end terminals, also referred to as mesh clients (MCs), are connected to special nodes, called mesh routers (MRs). These nodes do not generate traffic, since they are simply meant to relay the packets of their MCs. Additionally, some MRs, called mesh access points (MAPs), can be provided with cabled connection, and can therefore act as gateways toward the Internet. A MAP is also wirelessly interconnected to every other MR in a multihop fashion. In contrast, an MC can interact only with the MR to which it is connected. MRs form what is usually referred to as the backbone of the WMN, which can physically cover a large region using wireless multihop communication. A possible realization of a WMN is depicted in Fig. 1. This structure offers a good cost/benefit balance, since it almost entirely avoids cable setup. For this reason, it is deemed applicable in rural areas as well as dense residential or business areas, where the deployment of wireline networks may be too expensive or difficult because of physical obstacles.

The first hop from any MC to its related MR is often assumed to employ widespread cost-effective radio technologies (e.g., IEEE 802.11 [2]). The multihop communication among MRs is an open issue and involves several challenges related to different layers of the protocol stack. On one hand, the creation of low-interference high-rate paths to the MAPs is key to achieve good rates at each MR. On the other hand, the link layer needs to schedule packets over multiple links in order to achieve good transmission parallelism and possibly forward more data toward the MAPs at the same time. Find- ing the optimal path toward an MAP and scheduling links so as to maximize the transmission parallelism are traditionally performed by the routing algorithm running at the network layer and by the medium access control (MAC) protocol at the link layer, respectively. However, in a multihop wireless network, the routing algorithm needs to deal with link scheduling. If predefined routes (e.g., based on a shortest path criterion) are used, any scheduling algorithm will be forced to activate only the links belonging to that route. The combined result may be suboptimal in the sense that not all available network resources are utilized. In [3] the authors addressed the question of combining optimal link scheduling with suboptimal routing and vice versa, and pointed out that these tasks affect each other, and their optimality is strongly coupled. This is mainly due to the broadcast nature of the wireless medium, which combines the advantage of allowing each MR to communicate with multiple MRs through a single network interface with the disadvantage that simultaneous transmissions from different MRs may interfere with each other. Therefore, the main conclusion of [3] is that interference awareness available at the link layer must be exploited at the routing level.

Cross-layer design, which solves a joint routing and scheduling (JRS) optimization problem, is thus considered very promising to fully exploit WMN capacity. Recently, several papers have proposed solutions in this area. An example framework for JRS has been proposed in [4], where the authors introduce a heuristic technique to solve the crosslayer problem. In [5] JRS for multihop networks is presented, which also includes power control. The leitmotif of all the 


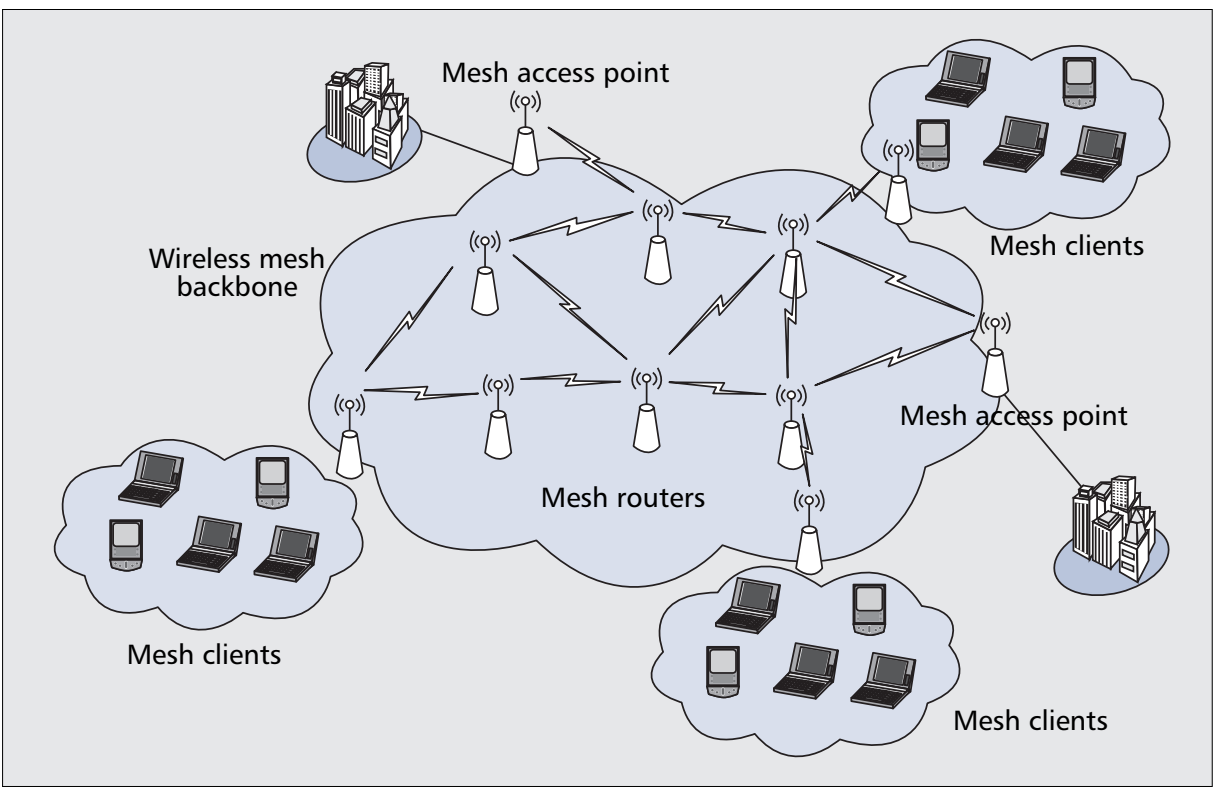

Figure 1. A possible structure for a wireless mesh network.

transmitting to $j$ at time $t$. To this end, we use a binary indicator variable $x_{i j}(t)$, equal to 1 if $(i, j)$ is active at time $t$ and 0 otherwise. The JRS problem corresponds to determining the pattern of link activations described by variables $x_{e}(t)$ as time goes by. To some extent, this addresses both scheduling and routing as the routes can be implicitly inferred by tracking subsequent link activations. For example, consider the WMN represented in Fig. 2. In this network a packet can be routed from $A$ to $\mathrm{F}$, say, by activating links $\mathrm{A} \rightarrow \mathrm{B}, \mathrm{B}$ $\rightarrow \mathrm{E}$, and eventually $\mathrm{E} \rightarrow \mathrm{F}$ at three separate time instants. These subsequent activations must be a packet transmission time apart from each other.

Furthermore, we speak of constraints to describe any limitation

papers described above is that approaching a JRS problem requires the specification of an interference model, which basically defines whether or not simultaneous transmissions of different MRs can be correctly decoded at their receivers. The most widely used classification of interference models in the literature dates back to [6], and distinguishes between the socalled physical and protocol interference models. In the former, the feasibility of simultaneous link activations is determined by the signal-to-interference ratio (SIR) of all receivers being above a given threshold. The latter instead imposes simpler interference conditions modeled through graph neighborhood relationships. The main problem of the existing approaches is that the proposed algorithms are strongly coupled with the interference model adopted. Thus, they may turn out to be suboptimal if more realistic models are considered.

In this article we present a general framework that decouples the notions of transceiver capability and wireless interference from the JRS algorithms. To study routing and scheduling under the graph formulation, we use the language of constrained linear programming problems, as commonly done in related work [4]. We represent the backbone of a WMN as a directed graph $G=(N, E)$. The nodes in set $N$ are the MRs, which are in turn connected by directed edges belonging to set $E$, which are ordered pairs of nodes and thus represent the communication links of the backbone. The link where a sender node $\mathrm{i} \in N$ transmits to a receiver $j \in N$ is represented by $(i, j)$, included in $E$ only if node $j$ can receive a transmission from $i$ in the absence of any other interference source. We also denote with $R_{i}$ and $S_{i}$ the set of nodes that are possible receivers from and senders to node $i$, respectively (i.e., the one-hop output and input neighbors of $i)$. Formally: $\boldsymbol{R}_{i}=\{j \in N:(i, j) \in E\}, S_{i}=\{j \in N:(j, i) \in E\}$. We also consider a parameter $g_{i j}$ corresponding to the wireless link gain when transmitting from $i$ to $j$. The rule for the inclusion of $(i, j)$ in $E$ can thus be that $g_{i j}$ is above a given threshold. Note that in realistic wireless scenarios, the performance of the forward and reverse links are not necessarily the same. Actually, the existence of the reverse link $(j, i)$ for every $(i, j) \in E$ is not even guaranteed. One notable exception in this sense is represented by WMNs using the IEEE 802.11 MAC on the backbone, since in this standard bidirectionality of links is required.

We speak of activation of link $e=(i, j)$ at time $t$ if $i$ is imposed on the activation of links by MAC and physical layers. In the rest of this article we discuss the constraints that must be satisfied for multiple transmissions to be feasible, and their impact on network performance. The analysis of these constraints is subdivided into two parts. First, we talk about the constraints involving the physical capabilities of the radio transceiver. This class of constraints is very general and independent of those due to wireless interference, and confusion between the two concepts should be avoided. Second, we describe the constraints specifically related to interference, also giving pointers to interference models present in the literature and referring to the MAC protocols specified in some common wireless interface standards that inspire the formulation of these constraints. Finally, we provide numerical insights into the performance of JRS in WMNs when different interference models are employed. To this end, we consider an underlying spaceand time-division multiple access (STDMA) scheme where JRS is performed.

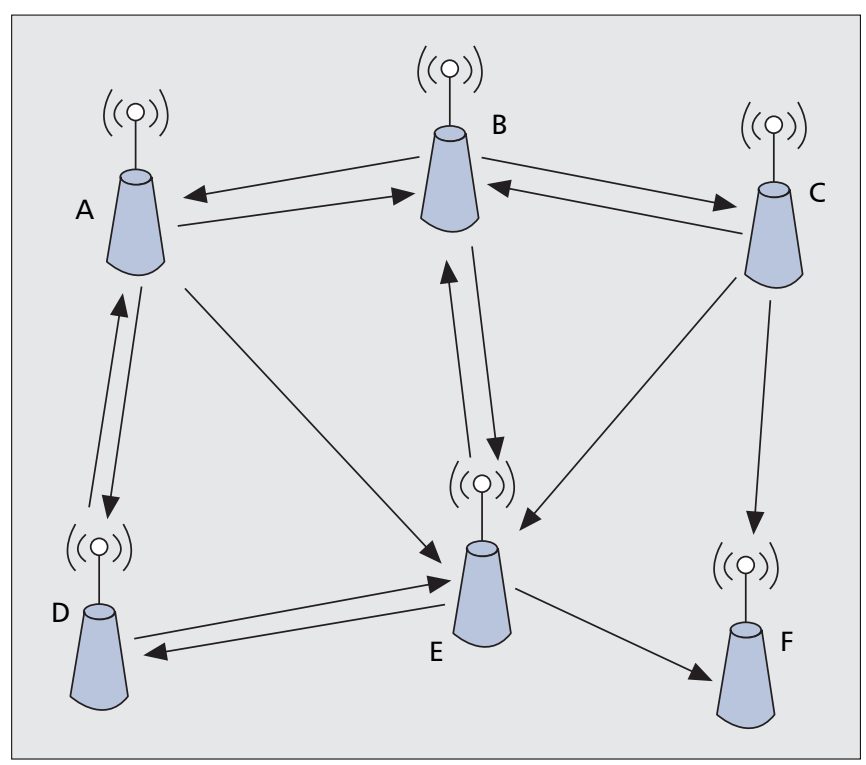

Figure 2. A sample wireless mesh network topology. 


\section{Wireless Transceiver Constraints}

The first constraint on link activation to solve JRS relates to the fact that the node capabilities for transmission and reception are limited. In particular, we focus on WMNs operating with a single omnidirectional antenna on narrowband channels, where it is not possible to receive simultaneously from multiple sources. Special techniques, such as wideband codedivision multiple access (WCDMA) or multiple-input multiple-output (MIMO) channels, can improve this condition. For simplicity, we do not investigate these issues; however, the simple model analyzed here can easily be extended to also take these cases into account. Therefore, we assume that at most one signal can be decoded, and any other transmission the receiver is able to hear can only be regarded as interference. The presence of interference at the receiver does not necessarily mean that the packet cannot be correctly decoded: the interference model comes into play at this point. However, regardless of the interference model, the maximum number of possible simultaneous successful receptions is one.

A similar situation happens for the transmitter. In particular, observe that we focus on unicast transmissions. Thus, even though the wireless medium is broadcast, and therefore the same transmission can be heard by many receivers, the message has only one intended destination. On the other hand, multiple transmissions from the same node are not possible. This assumption can be modified to account for cooperation among nodes [7] or network coding [8], which are not discussed here for space limitations, even though they are very promising research directions in wireless networks. For these reasons, we assume that a node can serve as the transmitter on at most one active link.

Finally, not only can simultaneous transmissions and receptions at the same node be at most one, but also the wireless communication medium is intrinsically half-duplex; that is, a node cannot listen on the same channel on which it is transmitting at the same time, or the transmitted power signal will jam any packet reception. Therefore, we impose the constraint of not activating more than one operation (i.e., either transmission or reception) for each node. Formally, this constraint translates into

$$
\forall i \in N, \forall t: \sum_{j \in S_{i}} x_{j i}(t)+\sum_{j \in R_{i}} x_{i j}(t) \leq 1
$$

The importance of constraint 1 is often underestimated when modeling multihop wireless networks. In fact, even the need for such a constraint is rarely mentioned. This may be due to mistaking it for an interference condition, whereas it refers to a physical limitation that holds irrespective of the interference model. To clarify this aspect, consider Fig. 2 again to check compatibility among link activations. The aforementioned transceiver constraint prevents, for example, simultaneous activation of links $\mathrm{A} \rightarrow \mathrm{B}$ and $\mathrm{A} \rightarrow \mathrm{D}$, since they share the same transmitter. Also, B $\rightarrow \mathrm{E}$ and $\mathrm{D} \rightarrow \mathrm{E}$ should not be activated together, as they share the same receiver; nor should $\mathrm{A} \rightarrow \mathrm{B}$ and $\mathrm{B} \rightarrow \mathrm{E}$, since $\mathrm{B}$ cannot receive and transmit simultaneously. Constraint 1 , instead, does not say anything about simultaneous activation, for example, of links $\mathrm{A} \rightarrow \mathrm{D}$ and $\mathrm{B} \rightarrow \mathrm{E}$, which involve entirely different pairs of nodes. However, due to the broadcast characteristic of wireless propagation, nodes can be reached by a transmission even though they are not the intended receiver, as happens, say, with transmission $\mathrm{A} \rightarrow \mathrm{D}$ also reaching $\mathrm{E}$. Thus, this kind of transmission is possibly limited by the wireless interference constraint, a further limitation described in the next section.

\section{Wireless Interference Constraint}

The usual classification of wireless interference models distinguishes between the protocol and the physical interference model [6]. Sometimes, other extensions of these models are introduced to represent further transmission aspects such as directional antennas, thresholds for the capture effect, and so on. An overview of these aspects can be found in [9].

The protocol interference model, in its original version, follows the rationale behind the IEEE 802.11 MAC, which models interference as causing collision, or the impossibility of correctly decoding a received packet if some neighboring nodes simultaneously exchange messages, disturbing the ongoing transmission. The main advantage of an interference description through the protocol model is its conceptual simplicity and the ease of mathematically formalizing the resulting interference conditions.

The rules of the protocol interference model impose the condition that when certain transmissions are assumed to cause collision, they are simply forbidden to be simultaneously activated. A way to formalize this constraint is to define, associated with any edge $e \in E$, a conflicting set $I(e) \subseteq E \backslash\{e\}$. The required condition is that if edge $e$ is active, $I(e)$ must contain no active edges. Formally,

$$
\text { if } x_{e}(t)=1, \quad \text { then } \sum_{f \in l(e)} x_{f}(t)=0 \text {. }
$$

In the literature, several possibilities have been presented to determine the set $I(e)$, all generically called the protocol interference model but presenting subtle differences. Our goal here is to propose a taxonomy for what we identify as a class of interference models, since it actually encompasses several mathematical formulations. Moreover, we aim to show the relationship, suggested by the name itself, with the MAC protocols possibly used in the WMN.

As a first step, we intentionally introduce a very simple member of the protocol interference model class, using the straightforward possibility $\mid(e)=E \backslash\{e\}$ for all $e \in E$; that is, at most one edge can be activated at any given time throughout the whole network. In other words, either exactly one edge is active, or no edge is active at all. Due to this property, we refer to this version as the 01protocol model. Even though it is quite oversimplified, this model can be useful as a theoretical term of comparison. In fact, the 01 protocol model takes the most conservative approach to interference protection, so space diversity is not exploited to obtain transmission parallelism. Actually, this situation necessarily occurs in certain special topologies. For example, in [9] this model is mentioned as used in [2] to derive the performance of the distributed control function (DCF) in an IEEE 802.11 hotspot controlled by a single access point. Indeed, it is true that the 01 protocol model holds here, but the reason is not electromagnetic interference, but rather that the topology is a star network (every node is connected only to the access point). Therefore, this situation is due to the wireless transceiver constraint, not to interference.

Apart from the 01 protocol model, other versions rely on propagation aspects, described with simplified geometric approaches. The idea is to define interference regions, areas of the physical space where ongoing transmissions interfere with each other and cannot coexist. We also remark that most of the existing work takes a very simplified approach where the interference region is modeled as a circular area with fixed range equal for all nodes. Within these regions, the rationale of the 01 protocol model is kept, so the limitation of having at most one active transmission is restricted to a small- 
er region. In the end this simply translates to a different (i.e., stricter) definition of the set $1(e)$.

In the following we implicitly assume that all propagation and interference aspects are again described through the graph $G=(N, E)$; not only can node $i \in N$ transmit to $j \in N$ if and only if $(i, j) \in E$, but also, $i$ can disturb other transmissions intended for $j$ from other nodes. Actually, the requirement of correct reception is generally more restrictive than pure disturbance, since it is possible to jam the reception of another node without being able to transmit to it. However, for simplicity we assume that the transmission range of a node equals its interference range. The analysis of the cases where this does not happen can be done in an entirely similar manner by considering additional virtual links not representing any real communication, just interference.

In the original and more common version, which we call hereafter the 11protocol model, it is implicitly assumed that IEEE 802.11 MAC is employed. Note that IEEE 802.11 is designed to work for bidirectional links only and heavily relies on this hypothesis: for IEEE 802.11-based networks, $(i, j) \in E$ if and only if $(j, i)$ also belongs to $E$. Following IEEE 802.11 MAC, the 11protocol model dictates that a transmission on $(i$, $j) \in E$ is interference-free and can therefore be activated only if there are no transmitters or receivers belonging to any active link that can disturb either $i$ or $j$. Note that the reason for requiring the absence of interferers in both the receiver's and transmitter's disturbance area of both interfering transmitters and receivers is that the IEEE 802.11 standard forces the receiver to acknowledge request to send (RTS) and data packets with clear to send (CTS) and acknowledgments (ACKs), respectively. In other words, during the ACK exchange, a logical receiver becomes a physical transmitter, so it can cause disturbance to others. Similarly, the logical transmitter needs to perform reception (of ACKs), for which it has to be collision-free. This also justifies the need for the existence of both forward and reverse links.

A possible definition of $I(e)$ in the 11 protocol model is thus

$$
\begin{aligned}
\mathrm{I}((i, j))= & \{(k, \ell) \in E,\{k, \ell\} \cap\{i, j\}=\varnothing: \\
& \{i, j\} \cap\left(R_{k} \cup R_{\ell}\right) \neq \varnothing .
\end{aligned}
$$

Clearly, this condition can be relaxed if the IEEE 802.11 MAC protocol is not used (e.g., if IEEE 802.16 is used instead). There are differences, not discussed here since they are out of the scope of this analysis, between the handshake procedures of IEEE 802.11 and IEEE 802.16, which change the relationships of interference among nodes. We can then formulate a 16protocol interference model, which proceeds identically to the 11 protocol model, with the notable exception that collision occurs only when the designated receiver is interfered by another transmitter. Any other combination (transmitter is under coverage of an interfering transmitter, or another receiver covers either the receiver or the transmitter) does not do any harm. This formally results in

$$
I((i, j))=\left\{(k, \ell) \in E,\{k, \ell\} \cap\{i, j\}=\varnothing: j \in R_{k}\right\} .
$$

The 16protocol model simplifies the 11 protocol model as it considers the receiver $j$ being under coverage of an interfering transmitter $k$ as the only situation where collision occurs. The 11 protocol model instead considers four possible combinations as colliding: all cases where $i$ or $j$ is under coverage of either an interfering transmitter $k$ or an interfering receiver $\ell$. In most papers dealing with WMN backbone management, the 11 protocol model is what is meant when the protocol model is cited. However, if links are not bidirectional and the MAC does not follow the IEEE 802.11 standard, there is no reason to use the 11 protocol model. If the MAC protocol does not require acknowledgment, the 16 protocol model would be more appropriate.

To sum up, the protocol interference model is easy to implement, and offers several possibilities to both describe MAC aspects, which have been classified in the three different versions (01protocol, 11protocol, 16protocol) and employ the preferred mathematical model (coverage/disturbance range, conflict graph, neighborhood relationships). However, these practical advantages come at the price of some theoretical drawbacks. In fact, all versions of the protocol model are imperfect in capturing wireless interference. First of all, the characterization of wireless propagation is not entirely realistic, especially if multiple power levels are adopted. Moreover, a definite criticism of the protocol model is that interference is not a binary relationship $[3,10]$.

For example, strong interference, which leads to packet loss, may be present if more than two specific edges are simultaneously activated, but not when any two of them are. Consider again Fig. 2: it is possible that $\mathrm{A} \rightarrow \mathrm{D}$ and $\mathrm{B} \rightarrow \mathrm{E}$ can be activated together if and only if $\mathrm{C} \rightarrow \mathrm{F}$ is not active simultaneously. Thus, the condition of interference cannot be translated into a binary relationship, as there is no specific link among $\mathrm{A} \rightarrow \mathrm{D}, \mathrm{B} \rightarrow \mathrm{E}$, and $\mathrm{C} \rightarrow \mathrm{F}$ that causes interference; the problem is the joint effect of all of them. The conflict set I(e), which must be evaluated pairwise, is not appropriate in this case.

These problems can be overcome by means of the physical interference model, whose rationale is as follows. The packet error rate (PER) at the receiver is a monotonically increasing function of the signal-to-interference-plus-noise ratio (SINR). It is often reasonable to consider a simplified threshold approach, where a packet is correctly received with probability 1 if the SINR is above a given threshold and always erroneous otherwise. Formally, the following condition must hold:

$$
\frac{P_{i} g_{i j}}{\Sigma_{k \neq i} P_{k} g_{k j}+N_{j}} \geq \gamma,
$$

where $(i, j)$ is the link of interest, the index $k$ in the sum denotes a possible interferer (the intended transmitter $i$ is excluded from the sum), $P_{x}$ is the power emitted by node $x$, and $N_{j}$ is the receiver noise at node $j$. It is not restrictive to take the value $\gamma$, which defines the SINR threshold, equal for all nodes.

Other assumptions, made only for ease of presentation but without loss of generality, as avoiding them would only lead to a more cumbersome (though conceptually identical) formulation, are as follows: we neglect the noise terms, and we consider an equal power level $P$ among all transmitting nodes. In particular, the last assumption is equivalent to assuming that the power level used by each node is constant over time. In this case any element $g_{i j}$ can be replaced by $g_{i j}^{\prime}=g_{i j} P_{i}$, thus omitting the power term. Otherwise, an extended framework, where power control is also considered, could be realized by following the rationale presented in [5].

In the context of our framework, which describes JRS through link activation patterns, the constraint can be formalized as follows:

$$
\text { if } x_{i j}(t)=1, \quad \text { then } g_{i j} \geq \gamma \sum_{k \in S_{j} \backslash\{i\}} g_{k j} \underset{\square \in R_{k} \backslash\{j\}}{\sum_{k} x_{k}(t) .}
$$

Reducing the PER function to a step function with transition value $\gamma$ is indeed an approximation. However, it is still much more accurate than the ones made under the protocol models, as it better takes into account physical propagation. 


\begin{tabular}{|c|c|c|}
\hline Class & Methodology & Mathematical relationship \\
\hline Protocol & $\begin{array}{l}\text { For each active link e, all links } \\
\text { in the set } I(e) \text { must be inactive }\end{array}$ & $\begin{array}{l}01 \text { protocol model: } \\
I((i, j))=E \backslash\{(i, j)\} \\
11 \text { protocol model: } \\
I((i, j))=\{(k, \ell) \in E,\{k, \ell\} \cap\{i, j\}=\varnothing:\}\left\{i, j \cap\left(R_{k} \cup R_{\ell}\right) \neq \varnothing\right\} \\
16 \text { protocol model: } \\
I((i, j))=\left\{(k, \ell) \in E,\{k, \ell\} \cap\{i, j\}=\varnothing: j \in R_{k}\right\} .\end{array}$ \\
\hline Physical & $\begin{array}{l}\text { For each active link } e \text {, the SIR } \\
\text { must be over the threshold } \gamma\end{array}$ & $g_{i j} \geq \gamma \sum_{k \in S_{j} \backslash\{i\}} g_{k j} \sum_{\ell \in \mathcal{R}_{k} \backslash\{j\}} x_{k \ell}(t)$ \\
\hline
\end{tabular}

Table 1. Taxonomy of interference models.

Moreover, as opposed to the collision assumption, in the physical model a correct packet reception is allowed even in the presence of (moderate) interference, and the cumulative character of interference is accounted for. Indeed, the choice of $\gamma$ depends on the shape of the PER function, which in turn relates to the modulation and coding scheme, and on the PER value, which is considered as acceptable at the application level. However, none of these factors depend on MAC issues; thus, the physical model allows operating between MAC and other layers in a more modular manner. The drawback of this model is that it translates into more complex mathematical relationships than the protocol model. Moreover, if a specific MAC needs to be addressed, additional constraints (e.g., related to acknowledgments) are required.

A taxonomy summarizing the mathematical formulations and the rationale behind each interference model is reported in Table 1. We remark again that the choice of the model ultimately depends on the purpose of the analysis. The protocol models offer better conceptual simplicity and offer an easy way to represent network constraints. However, the physical model has a good point against the protocol model, as described in [10], when the analysis comes down to the achieved performance of JRS in a WMN. This aspect is further investigated in the next section by means of numerical examples.

\section{Performance Evaluation}

In this section we focus on the JRS problem of defining efficient link activation patterns that not only satisfy all the constraints but also efficiently deliver traffic to the MAPs acting as gateways for the WMN. We consider discrete (slotted) time, where a time slot is equal to the packet transmission time (assumed, for simplicity, the same for all nodes). We focus on the minimal time scheduling problem: to deliver a given amount of traffic from all non-gateway MRs to the MAPs in the shortest possible time. With minor modifications, our framework can work to solve other problems as well, for example, where the goal is throughput maximization (obtaining the highest amount of traffic delivered to the gateways within an assigned time) or fairness and/or prioritization among the traffic from different MRs is taken into account.

For simplicity, we assume that the initial backlog of each MR is known a priori, and no further packet arrivals take place after link activation has started. Solutions that take into account variations of the estimated traffic from each MR can also be envisaged, which is an interesting direction for future research. To evaluate the impact of the chosen interference model on the performance results, we have implemented our framework to verify the transmissions' compatibility providing support for all the interference models described in this arti- cle. We stress that the derivation of efficient algorithms to solve the minimal time scheduling problem is out of the scope of this article. Here, we have computed within a simulator the optimal link schedule with an exhaustive search over all possible link activation patterns that are feasible according to the constraints, in particular to the specific interference model employed.

We consider a grid topology, represented in Fig. 3, consisting of $30 \mathrm{~m} \times 30 \mathrm{~m}$ squares. Nodes occupy the grid intersections in a contiguous manner. We consider $2 \times 3,3 \times 3,3 \times 4$, and $4 \times 4$ grid placements of the nodes. We assume that there is only one MAP in the network (placed in a corner of the grid), and each of the other MRs has 12 packets to transmit toward the gateway. Wireless propagation is modeled by considering a simple path loss expression proportional to $d^{-4}$, where $d$ is the sender-receiver distance. A link $(i, j)$ is included in $E$ when its gain $g_{i j}$ is higher than $-60 \mathrm{~dB}$ with respect to the attenuation at $1 \mathrm{~m}$. This results in a simple scheme where all nodes can only communicate with their physical one-hop neighbors on the grid. However, we stress that the validity of the conclusions drawn in the following holds for any scenario and also when more complicated propagation models are used to determine the $g_{i j}$ parameters.

In Figs. 4 and 5, we report the length of the optimal schedule computed when either protocol or physical interference models are adopted, respectively. In both figures we also

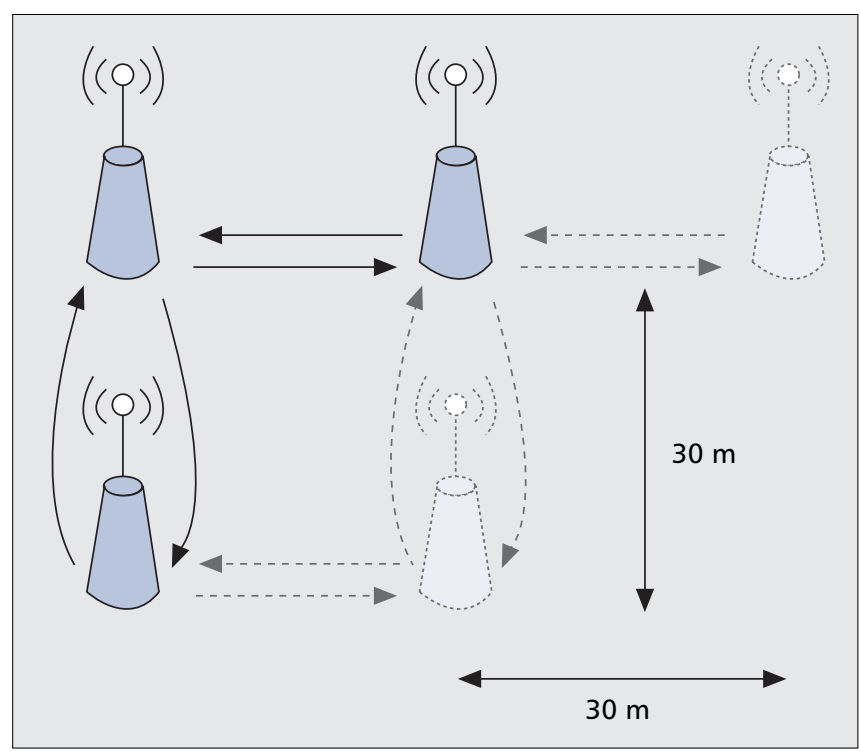

Figure 3. Grid topology considered for the numerical evaluations. 


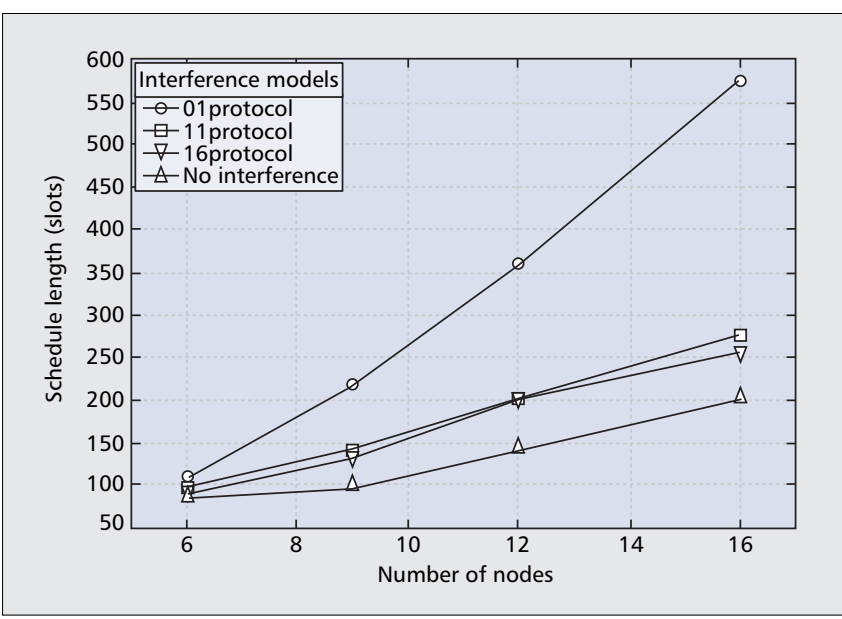

Figure 4. Optimal schedule length with different protocol interference models.

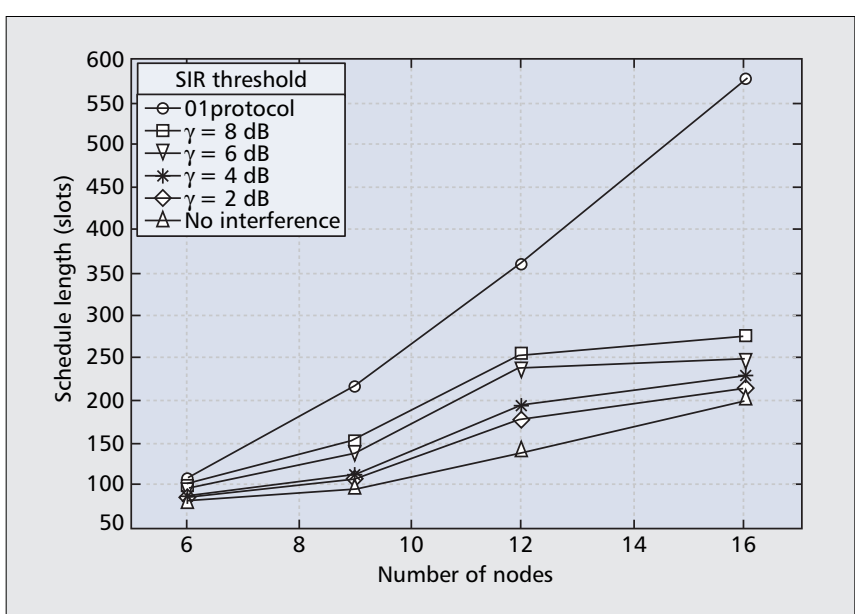

Figure 5. Optimal schedule length with a physical interference model with different values of SIR target. report the results for the 01 protocol model, where at most one link can be active at a time, and for no interference (i.e., only the transceiver constraints are considered; SIR threshold $\gamma$ equal to 0$)$. The former provides an upper bound to the length of the schedule as in this case no simultaneous transmissions are permitted, whereas the latter gives a lower bound since maximum transmissions parallelism is guaranteed. As can be seen in Fig. 4, the length of the schedule increases (almost) linearly with the number of nodes in the grid in all cases, with a slope that depends on the specific model. Both the 11 protocol and the 16 protocol curves lie within the 01 protocol and no interference cases. As expected, the more stringent the constraints of a model, the longer the duration of the corresponding schedule. For example, the curve corresponding to the 11 protocol model always lies above that for the 16 protocol. The gap between these curves can be significant in terms of slots with a maximum of about 30 slots in the case of a $4 \times 4$ grid (16 nodes).

As far as the physical model is concerned, in Fig. 5 the curves correspond to different values of the SIR threshold $\gamma$ ranging from 2 to $8 \mathrm{~dB}$. Also for the physical model, in general, the length of the schedule increases with the number of nodes. Furthermore, since increasing the SIR threshold reduces the transmission parallelism, the resulting schedule length increases with the value of the SIR threshold. Note that adopting the physical interference model can provide results that are closer to the no interference curve than those obtained by any protocol model. Moreover, by varying the SIR threshold, we can obtain a wide range of possibilities that permit various performance levels tunable at a fine grain. For example, with $\gamma$ equal to $6 \mathrm{~dB}$ we basically obtain the same results as for the 16 protocol, whereas with $\gamma$ equal to $2 \mathrm{~dB}$ the curve is quite close to the no interference case.

Beyond the numerical analogies, the most important consequence is that the physical interference model definitely allows a higher degree of tunability and better capability of capturing wireless interference. Apart from being grounded on a stronger theoretical basis, the physical model also allows us to change, by simply varying the parameter $\gamma$, the entire performance characterization. The protocol models are instead stuck on a single value. Moreover, we remark that the trend shown for relatively higher network sizes exhibits a steeper increase of the schedule length for the protocol models (Fig. 4) rather than for the physical model (Fig. 5). This is again because the cumulative character of the interference becomes more relevant, and the protocol model, which accounts for it in a more conservative manner, (unnecessarily) decreases the network parallelism even more. Thus, using the physical model allows higher scalability, since it gives a more accurate evaluation of the schedule length when the number of nodes grows, whereas the protocol models further degrade the performance.

To improve the protocol models, one should complicate them by introducing an interference range separate from the transmission range and modeling the so-called capture effect. On the other hand, we remark that these characteristics are already present in the physical interference model and can be obtained by simply tuning the parameter $\gamma$, which corresponds to a more sensible process as it directly relates to physical quantities. The case where interference can be tolerated is particularly critical, as all the protocol models (even the best, i.e., the 16protocol model) have a significant gap from the no interference case, whereas the physical model can correctly represent this situation with a low value of $\gamma$.

\section{Conclusions}

The impact of interference on JRS is very strong, and its correct characterization is key for a sensible network analysis. The choice of the right interference model depends on many factors, including ease of implementation and the possibility of obtaining realistic results. In this sense, there is no definitive choice among the models presented here. We remark, however, that the widespread use of protocol interference models adopts nonuniform notations that need to be harmonized. In this sense, our taxonomy aims to clarify the differences among the possible choices.

Moreover, all the protocol models lead to performance limitations, since they are very restrictive and obtain lower network parallelism than the physical model due to their requirement of silencing allegedly colliding connections. This does not hold only for the 01 protocol model, which is easily seen to be unrealistic, since the widely used 11 protocol and 16 protocol model also suffer from similar problems.

This results in an undesirable lack of parallelism for WMNs, which are meant to provide good network coverage and high data rates network-wide. The more accurate characterization of the system obtained by utilizing the physical model reveals interesting behaviors, which may lead to reconsidering the design criteria of access protocols for WMN. Although the mesh versions of both IEEE 802.11 and IEEE 802.16 take these aspects into account, the protocol design of improved interference-aware JRS strategies is still an open research challenge. 


\section{References}

[1] I. F. Akyildiz, X. Wang, and W. Wang, "Wireless Mesh Networks: A Survey," Computer Networks, vol. 47, Mar. 2005, pp. 445-87.

[2] G. Bianchi, "Performance Analysis of the IEEE 802.11 Distributed Coordination Function," IEEE JSAC, vol. 18, no. 3, 2000, pp. 535-47.

[3] K. Jain et al., "Impact of Interference on Multi-Hop Wireless Network Performance," Wireless Networks, vol. 11, no. 4, July 2005, pp. 471-87.

[4] M. Kodialam and T. Nandagopal, "Characterizing Achievable Rates in MultiHop Wireless Mesh Networks with Orthogonal Channels," IEEE/ACM Trans. Net., vol. 13, no. 4, Aug. 2005, pp. 868-80.

[5] R. L. Cruz and A. V. Santhanam, "Optimal Routing, Link Scheduling and Power Control in Multihop Wireless Networks," Proc. IEEE INFOCOM, vol. 1, San Francisco, CA, 2003, pp. 702-11.

[6] P. Gupta and P. R. Kumar, "The Capacity of Wireless Networks," IEEE Trans. Info. Theory, vol. 46, Mar. 2000, pp. 388-404.

[7] A. Sendonaris, E. Erkip, and B. Aazhang, "User Cooperation Diversity-Part I: System Description," IEEE Trans. Commun., vol. 51, no. 11, Nov. 2003, pp. 1927-38.

[8] R. Ahlswede et al., "Network Information Flow," IEEE Trans. Info. Theory, vol. 46, no. 4, July 2000, pp. 1204-16.

[9] A. lyer, C. Rosenberg, and A. Karnik, "What is the Right Model for Wireless Channel Interference?", Proc. QShine, no. 2, Waterloo, Ontario, Canada, 2006.

[10] G. Brar, D. Blough, and P. Santi, "Computationally Efficient Scheduling with the Physical Interference Model for Throughput Improvement in Wireless Mesh Networks," Proc. ACM MobiCom, Los Angeles, CA, Sept. 2006, pp. 2-13.

\section{Biographies}

LEONARDO BADIA [M'04] (leonardo.badia@imtlucca.it) received a Laurea degree (with honors) in electrical engineering and a Ph.D. in information engineering from the University of Ferrara, Italy, in 2000 and 2004, respectively. During 2002 and 2003 he was on leave at the Radio System Technology Labs (now Wireless@KTH), Royal Institute of Technology, Stockholm, Sweden. After having been with the Engineering Department of the Universita di Ferrara, Italy, in 2006 he joined the "Institutions, Markets, Technologies" (IMT) Lucca Institute for Advanced Studies, Lucca, Italy, where he is currently a research fellow. He also collaborates with DEI, University of Padova, Italy. His research interests include energy-efficient ad hoc networks, transmission protocol modeling, admission control, and economic modeling of radio resource management for wireless networks. He serves as a reviewer for several periodicals in communications.

ALESSANDRO ERTA (alessandro.erta@imtlucca.it) graduated (cum laude) in computer systems engineering from the University of Pisa, Italy, in February 2005. He is currently a Ph.D. student at IMT Lucca Institute for Advanced Studies. In 2006 he was a visiting student at Rice University, Houston, Texas. Currently, he is spend- ing the last part of his Ph.D. program as an R\&D project manager with Fluidmesh Networks, Inc. developing solutions for wireless mesh networks. He has been involved in national projects as well as research projects supported by private industries. His research interests include quality of service in wireless networks, design and performance evaluation of MAC protocols, and scheduling algorithms for wireless mesh networks.

LUCIANO LENZINI (I.lenzini@iet.unipi.it) received a degree in physics from the University of Pisa. He joined the Centro Nazionale Universitario di Calcolo Elettronico (CNUCE), an institute of the Italian National Research Council (CNR), in 1970. In 1994 he joined the Department of Information Engineering of the University of Pisa as a full professor. His current research interests include the design and performance evaluation of MAC protocols for wireless networks, architectures and protocols for mesh networks, and quality of service provision in integrated and differentiated services networks. He is currently on the editorial boards of Computer Networks and the Journal of Communications and Networks as area editor for wireless networks. He served as general chairman for the 1992 IEEE Workshop on Metropolitan Area Networks, 2002 European Wireless Networks, and the 2006 International Conference on Performance Evaluation Methodologies and Tools (ValueTools), of which he is a co-founder. He has been involved in several national and international projects in the area of computer networking.

MICHELE ZORZI [F'07] (zorzi@dei.unipd.it) received a Laurea degree and a Ph.D. in electrical engineering from the University of Padova, Italy, in 1990 and 1994, respectively. During 1992-1993 he was on leave at the University of California, San Diego (UCSD), attending graduate courses and doing research on multiple access in mobile radio networks. In 1993 he joined the faculty of the Dipartimento di Elettronica e Informazione, Politecnico di Milano, Italy. After spending three years with the Center for Wireless Communications at UCSD, in 1998 he joined the School of Engineering of the Universita di Ferrara, Italy, where he became a professor in 2000. Since November 2003 he has been on the faculty at the Information Engineering Department of the University of Padova. His present research interests include performance evaluation in mobile communications systems, random access in mobile radio networks, ad hoc and sensor networks, energy constrained communications protocols, and broadband wireless access. He was Editor-InChief of IEEE Wireless Communications, 2003-2005, is currently the Editor-In-Chief of IEEE Transactions on Communications, and serves on the editorial boards of IEEE Transactions on Wireless Communications, Wiley's Journal of Wireless Communications and Mobile Computing, and ACM/URSI/Kluwer Journal of Wireless Networks. He was also guest editor for special issues of IEEE Personal Communications (Energy Management in Personal Communications Systems) and IEEE Journal on Selected Areas in Communications (Multimedia Network Radios). 